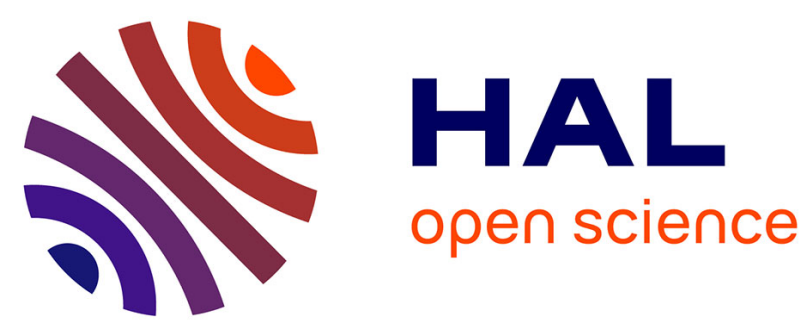

\title{
Le glycogène au cours de la formation des spermatozoïdes et de leur transit dans les tractus génitaux mâle et femelle chez le guppy (poisson Poecilide)
}

Roland Billard, Bernard Jalabert, Denise Guene, Anne-Marie Escaffre

\section{To cite this version:}

Roland Billard, Bernard Jalabert, Denise Guene, Anne-Marie Escaffre. Le glycogène au cours de la formation des spermatozoïdes et de leur transit dans les tractus génitaux mâle et femelle chez le guppy (poisson Poecilide). Annales de biologie animale, biochimie, biophysique, 1973, 13 (3), pp.313-320. 10.1051/rnd:19730301 . hal-00896773

\section{HAL Id: hal-00896773 https://hal.science/hal-00896773}

Submitted on 1 Jan 1973

HAL is a multi-disciplinary open access archive for the deposit and dissemination of scientific research documents, whether they are published or not. The documents may come from teaching and research institutions in France or abroad, or from public or private research centers.
L'archive ouverte pluridisciplinaire HAL, est destinée au dépôt et à la diffusion de documents scientifiques de niveau recherche, publiés ou non, émanant des établissements d'enseignement et de recherche français ou étrangers, des laboratoires publics ou privés. 
Ann. Biol. anim. Bioch. Biophys., I973, 13 (3), 313-320.

\title{
LE GLYCOGENE AU COURS DE LA FORMATION DES SPERMATOZOÏDES ET DE LEUR TRANSIT \\ DANS LES TRACTUS GÉNITAUX MÂLE ET FEMELLE CHEZ LE GUPPY (POISSON POECILIDE)
}

\author{
R. BILLARD et B. JALABERT \\ avec la collaboration technique de Denise Guene et Anne-Marie Escaffre \\ Laboratoire de Physiologie des Poissons, \\ Centre national de Recherches zootechniques, I. N.R.A. \\ 78350 Jouy en Josas
}

\section{RÉSUMÉ}

Des particules de glycogène $\beta$ et $\alpha$ ont été observées en grande quantité dès le début de la spermiogenèse dans les cellules de Sertoli et les spermatides au cours de la spermiogenèse. Ce glycogère se localise autour des mitochondries de la pièce intermédiaire. La présence de ce glycogène peut être mise en relation avec le type de spermiogenèse qui se déroule dans des cystes, ce qui interdit toute libération de polysaccharides dans le fluide testiculaire. La forte diminution des quantités de glycogène observée dans certains spermatozeugmes du tractus génital mâle et dans les spermatozoïdes stockés dans l'ovaire peut être interprétée comme résultant, soit de processus de vieillissement, soit de l'épuisement des réserves de glycogène au cours de la nage. Dans ces conditions, la présence de glycogène dans le spermatozoïde d'une espèce de Poisson à fécondation interne, pourrait être considérée, du fait de l'absence de liquide séminal, comme une source endogène de matériel énergétique.

\section{INTRODUCTION}

Les spermatozoïdes d'un grand nombre d'espèces d'Invertébrés et de Vertébrés inférieurs possèdent du glycogène au niveau de la pièce intermédiaire. Les premières démonstrations qui ont été fondées sur des caractères purement morphologiques, selon Drochmans (I962) ou cytochimiques, sont dues à PERsonNe et ANDRÉ (I964); LANZA et Quatririni (I964); Silveira et PorTer (I964); Von BonsDorfF et TElKKa (I965) ; LANZA (I965); HoRstmanN (I968). La mise en œuvre de la méthode 
de Threry (I967) pour la démonstration des polysaccharides (Personne, I968; Anderson, I968; Georges, I969; Giustr, I969; Anderson et Personne, i97o) a confirmé les premières observations.

Dans le testicule, l'existence de glycogène a été signalée dans les tubules séminifères (cf. MANN, I964). Des particules de glycogène ont été localisées dans les cellules de Sertoli des Poissons (Tilapia, Nichol,s et Graham, r972), des Amphibiens (Discoglosse, SANdoz, I970 ; Lézard, Dufaure, I97I) et de certains Mammifères : Cobaye (NICANDER, I957) ; Hamster (FoUQUET, I968) mais pas chez la Souris (NICANDER, I957 ; SANDOZ, I970). I1 n'a pas été démontré de glycogène dans les cellules germinales de Mammifères, qu'il s'agisse de spermatides (FouQuET, r968) ou de spermatozoïdes (ANDERson et Personne, r97o a). Dans le testicule de Hamster, seules les cellules de Sertoli peuvent métaboliser le glycogène (FoUQUET et GuHA, I969).

Chez le Guppy (Poecilia reticulata) le spermatozoïde possède de grandes quantités de glycogène (ANDERson et PERSONNE, I970 $a$ et $b$; BILIARD et BRETON, I970) mais l'origine n'en a pas été étudiée.

Le présent travail traite de cette origine du glycogène des spermatozoïdes (élaboration au cours de la spermiogenèse) et son devenir après transit dans les voies génitales mâles et femelles.

\section{MATÉRIEL, ET MÉTHODES}

Les Guppies (Poecilia reticulata PETERs) des deux sexes proviennent de la souche du laboratoire élevée dans les conditions déjà décrites (BILLARD, I969a).

\section{Fixation et inclusion}

Après anesthésie, les animaux sont sacrifiés et les gonades fixées par le glutaraldéhyde en solution à 3,3 ou $4 \mathrm{p}$. I oo dans un tampon phosphate $0,05 \mathrm{M}$ à pH 7,25 pendant $60 \mathrm{mn}$ à $4^{\circ} \mathrm{C}$. Un certain nombre de préparations subissent une double fixation dans une solution d'acide osmique à $2 \mathrm{p}$. Ioo dans le même tampon pendant $30 \mathrm{mn}$.

L'inclusion est faite dans l'épon selon Finck (1960).

\section{Mise en évidence du glycogène}

- Acide periodique-thiocarbohydrazide-protéinate d'argent (AP-TC-PA, THIERY, I967).

La technique de coloration complète ainsi que les colorations témoins sont résurrées dans le tableau I.

- Augmentation du contraste du glycogène par le plomb.

Les colorations sont conduites dans les conditions habituelles, acétate d'uranyle (5 mn), mais la durée de séjour dans une solution saturée d’hydroxyde de plomb est prolongée pendant $30 \mathrm{mn}$.

Les coupes sont recouvertes d'un film de carbone et observées à l'Elmiscope I Siemens.

La recherche du glycogène a été réalisée dans les cas suivants : I970) ;

- au cours de la spermiogenèse dont les stades ont été décrits précédemment (BILLARD,

- dans les spermatozoïdes du canal testiculaire et du canal déférent;

- dans les spermatozoïdes présents dans le tractus génital femelle.

L.es spermatozoïdes qui peuvent survivre plusieurs mois dans l'ovaire de la femelle (WINGE, I937; PlOYE, I969) sont examinés dans leur site de conservation (JALABERT et BILlARD, I969) chez 3 femelles adultes élevées en présence de mâles depuis leur naissance. 


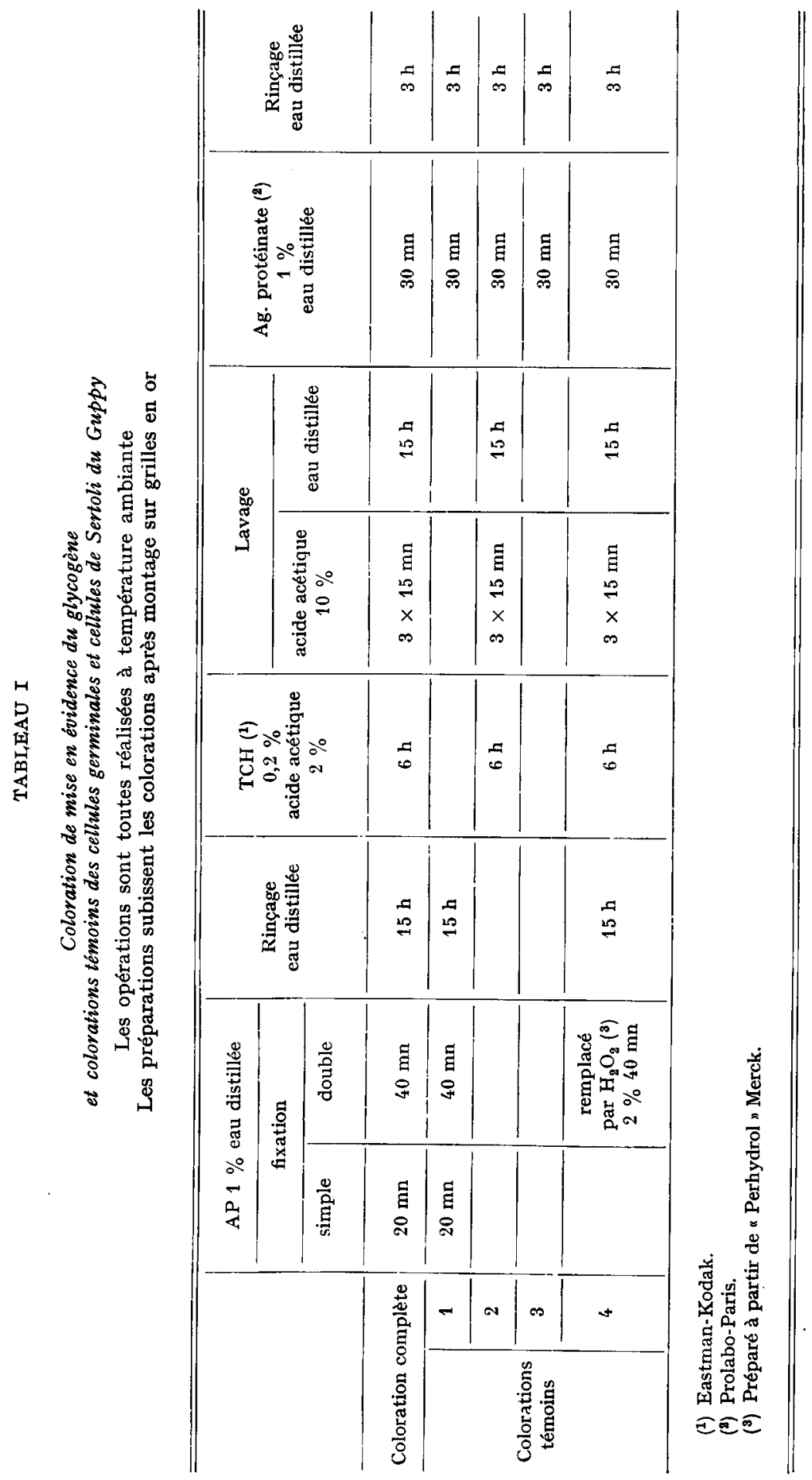




\section{OBSERVATIONS}

Les deux techniques d'identification mises en ouvre permettent de détecter des quantités importantes de glycogène dans le testicule du Guppy, mais seule, 1a technique AP-TC-PA qui donne les meilleurs résultats sera retenue dans les descriptions qui vont suivre. Dans tous les cas les colorations témoins sont négatives.

\section{Teneur en glycogène des cellules de Sertoli}

Dans les cellules de Sertoli des cystes à spermatogonies et à spermatocytes, le glycogène n'existe qu'à l'état de trace, mais il devient beaucoup plus abondant au début de la spermiogenèse ( $\mathrm{pl}$. I $\mathrm{A}$, III $\mathrm{A}$ ).

Il se présente toujours sous la forme de particules $\beta$ qui se répartissent de façon uniforme dans le cytoplasme. Le glycogène subsiste ainsi dans les cellules de Sertoli pendant toute la durée de la spermiogenèse. Des particules de glycogène existent également dans l'endothélium des vaisseaux sanguins du testicule (pl. III A)

\section{Teneur en glycogène des cellules germinales}

Spermatogonies et spermatocytes.

Des traces de glycogène sous forme de particules existent dans quelques spermatogonies et spermatocytes, mais les quantités sont très faibles.

\section{Spermatides.}

$A \mathfrak{u}$ début de la spermiogenèse, le glycogène n'existe dans les spermatides rondes $S_{1 a}$ qu'à l'état de trace toujours sous la forme de particules $\beta$ (pl. I A, I B). Le glycogène apparaît ensuite en quantités plus importantes dans les spermatides rondes $\mathrm{S}_{1 b}$ (pl. I B, II A).

Dans les spermatides, les particules de glycogène tendent à se localiser dans le voisinage des mitochondries où les particules $\beta$ se condensent partiellement en particules $\alpha$. Le glycogène reste à proximité des mitochondries et accompagne ces dernières lors de leur migration autour du flagelle (p1. II A). L'ensemble des îlots de glycogène se trouve rassemblé entre les mitochondries (pl. III B) ou sur la face interne du manchon mitochondrial (pl. II B) dès la phase $S_{2}$. Cette localisation périmitochondriale subsiste au cours des dernières phases de la spermiogenèse. Les résidus cytoplasmiques non expulsés à la fin de la spermiogenèse et qui subsistent dans les spermatozoïdes engagés dans le canal déférent, possèdent également des particules de glycogène $\alpha$ (pl. V). Les granules osmiophiles, abondants pendant la spermiogenèse ( $\mathrm{pl}$. II C) ne présentent pas la réaction caractéristique des polysaccharides (pl. II B).

Les spermatozoìdes du tractus génital mâle (p1. IV et V).

La quantité de glycogène est très importante dans les spermatozoïdes, entre les mitochonđries et dans le manchon cytoplasmique (p1. VII A) mais également 
autour du complexe centriolaire ( $\mathrm{pl}$. VI A). Les colorations témoins (pl. VI C et VI D) sont négatives. Dans la plupart des cas, tous les spermatozoïdes d'un spermatozeugme possèdent du glycogène, cependant quelques spermatozeugmes par testicule présentent des spermatozoïdes à peu près totalement dépourvus de glycogène (pl. IV).

\section{Les spermatozoides du tractus génital femelle.}

Tous les spermatozoïdes examinés dans le tractus génital femelle présentent une diminution considérable de la quantité de glycogène par rapport aux spermatozoïdes observés dans le tractus génital mâle ou dans les spermatozeugmes fraîchement éjaculés. Le glycogène ne subsiste qu'en faible quantité dans le manchon cytoplasmique et entre les mitochondries. Cette diminution du glycogène apparaît aussi bien sur coupe transversale de spermatozoïdes (p1. VII B) que sur coupe longitudinale (pl. VI B).

\section{DISCUSSION}

\section{Origine au glycogène dans le spermatozoïde}

Le glycogène déjà observé dans le spermatozoïde de Guppy (ANDERson et Personne, I970 $a$ et $b$; BirLaRd et BRETON, I970) s'élabore très tôt au cours de la spermiogenèse. Il apparaît dans les jeunes spermatides $S_{1 b}$ et se localise très rapidement au niveau de la future pièce intermédiaire. Le glycogène existe également dans les cellules de Sertoli mais seulement dans celles des cystes en spermiogenèse. Le glycogène apparaît d'ailleurs simultanément dans les cellules de Sertoli et dans les spermatides. Ceci pose le problème des relations entre le glycogène de ces deux types de cellules ainsi que celui de l'origine du glycogène dans les cellules germinales.

Il est d'autre part intéressant de souligner l'absence de glycogène dans les cystes en début de spermatogenèse (spermatogonies et spermatocytes) et son apparition dans les cystes qui entrent en spermiogenèse. Cette constatation traduit l'existence d'un changement de métabolisme qui affecte à ce stade aussi bien les cellules germinales que somatiques.

Les relations entre le glycogène des cellules de Sertoli et celui des cellules germinales, peuvent être considérées sous un angle topographique; du fait de leur organisation en cystes, les métabolites que reçoivent les cellules germinales doivent nécessairement transiter au travers des cellules de Sertoli à la périphérie du cyste. Il est cependant difficile d'imaginer un passage du glycogène sous forme figurée entre cellules de Sertoli et spermatides, bien qu'il existe des particules de glycogène extracellulaire à l'intérieur du cyste. Il est plus vraisemblable que le glvcogène sertolien lui-même originaire des vaisseaux sanguins soit transféré aux spermatides sous forme d'oligosaccharides. Il faut noter que les cellules de Sertoli du Guppy, du fait de leur localisation originale à la périphérie des cystes, ne peuvent pas participer directement à l'élaboration du fluide testiculaire comme elles peuvent le faire dans d'autres groupes de Poissons (BILLARD et al., I972) et chez les Mammifères (SETChELl et WaITES, I97I). Chez le Hamster, Fouquet (I968) a suggéré que le glycogène qui ne se trouve que dans les cellules de Sertoli et non dans les cel- 
lules germinales est libéré dans ce fluide testiculaire d'où il peut participer à la "nutrition " des spermatozoïdes. On peut supposer que chez le Guppy, en 1'absence presque complète de fluide testiculaire et de liquide séminal, le glycogène, après libération des cellules de Sertoli et réincorporation dans les spermatides, accompagne le spermatozoïde par une autre voie.

\section{Devenir et signification du glycogène dans le spermatozoide de Guppy}

La quantité de glycogène est toujours importante et constante dans les spermatozoïdes des cystes qui s'engagent dans le canal défférent. Au contraire, dans certains spermatozeugmes du canal testiculaire ou du canal déférent (p1. IV), les spermatozoïdes sont presque totalement dépourvus de glycogène. Cette perte de glycogène est donc intervenue au cours du transit dans les voies génitales mâles, transit dont la durée est très variable (BILIARD, I969 b). On pourrait donc supposer que les spermatozeugmes dépourvus de glycogène sont les plus âgés. Les spermatozoïdes examinés dans les voies génitales femelles sont aussi très appauvris en glycogène. Les spermatozoïdes présents dans l'ovaire peuvent être aussi très âgés, plusieurs mois selon WINGE (I937), de sorte que la disparition du glycogène pourrait être attribuée à un facteur temps et à des processus de vieillissement. Mais comme les spermatozoïdes des spermatozeugmes testiculaires présentent une légère agitation et comme les spermatozoïdes après accouplement doivent obligatoirement nager pour atteindre l'ovaire, on peut aussi considérer que la diminution du glycogène résulte de son utilisation en tant que fournisseur d'énergie dans les processus de motilité. Une telle hypothèse est supportée par 1'existence conjointe de glycogène et d'enzyme de son métabolisme dans de nombreux spermatozoïdes (ANDERson et Personne, i97o $a$ et $b$; Personne, et Anderson, I970).

Dans ces conditions, il serait possible qu'en l'absence de liquide séminal, mentionné précédemment, le glycogène constitue une source endogène de matériel glycolysable pour ce spermatozoïde qui doit assurer une fécondation interne.

Dans 1'hypothèse d'un intervention directe du glycogène sur la motilité, 1'épuisemment prématuré de certains spermatozoïdes observés dans le tractus génital mâle, pourrait avoir des conséquences importantes sur la fécondation. Ces considérations sont à rapprocher de la diminution du pourcentage de fécondation observé après insémination des femelles vierges avec des spermatozoïdes prélevés tous les mois par rapport aux fécondations obtenues avec des spermatozoïdes dont la fréquence de prélèvement est plus faible : 2 et 7 jours (BILLARD, I966). Il y a donc lieu de considérer cet aspect qualitatif des spermatozoïdes qui a été soulevé dans d'autres espèces de Poissons (Hochman, I966). Les données quantitatives sont donc insuffisantes pour rendre compte de l'efficacité de la spermatogenèse laquelle devrait également tenir compte de la notion de qualité des spermatozoïdes.

Reçu pour publication en février 1973.

\section{REMERCIEMENTS}

Ce travail a été réalisé en partie grâce à l'aide de la D. G. R. S. T. (contrat no 7 x.7.3027) et de l'Institut national de la Recherche agronomique. 


\section{SUMMARY}

\section{GLYCOGEN DURING FORMATION AND TRANSPORT OF SPERMATOZOA IN THE MALE AND FEMALE, GENITAL, TRACTS OF THE GUPPY (POECILID FISH)}

From the beginning of spermiogenesis, $\beta$ - and $\alpha$-glycogen particles are observed in the Sertoli cells and spermatids during spermiogenesis. The glycogen is localized around the mitochondria of the mid piece. The presence of this glycogen may be related to the type of spermiogenesis occurring in cysts, which prevents any polysaccharide release in the testicular fluid. The high decrease of the amount of glycogen observed in some spermatozeugma of the male genital tract and in spermatozoa stored in the ovary, may be interpreted as a result either of aging or of depletion of glycogen reserve while swimming. In these conditions, the presence of glycogen in the spermatozoon of a species of fish having internal fertilization, may be considered as an endogenous source of energy because the seminal fluid is absent.

\section{RÉFÉRENCES BIBLIOGRAPHIQUES}

ANDERson W. A., I968. Cytochemistry of sea urchin gametes. I. Intramitochondrial localization of glycogen glucose-6-phosphatase activity in spermatozoa of Paracentratus lividus. J. Ultrastruct. Res., 24, 398-4II.

Anderson W. A., Personne P., r97o $a$. The localization of glycogen in the spermatozoa of various invertebrate and vertebrate species. J. Cell. Biol., 44, 29-5I.

Anderson W. A., Personne P., r970 $b$. Recent cytochemical studies on spermatozoa of some invertebrate and vertebrate species. Comp. Spermat. B. Baccetti Ed., Academia dei Lincei, Rome, 43 I-449.

BILlard R., I966. Contribution à l'étude de la reproduction chez le Poisson Téléostéen Lebistes reticulatus au moyen de l'insémination artificielle. Thèse Biol. appl., Lyon, $83 \mathrm{p}$.

Billard R., I969a. La spermatogenèse de Poecilia reticulata. I. Estimation du nombre de générations goniales et rendement de la spermatogenèse. Ann. Biol. anim. Bioch. Biophys., 9, $25 \mathrm{r}-27 \mathrm{r}$.

Billa.AR R., I $969 b$. La spermatogenèse de Poecilia reticulata. II. La production spermatogénétique. Ann. Biol. anim. Bioch. Biophys., 9, 307-313.

Billard R., I97o. Ultrastructure comparée de spermatozoïdes de quelques poissons Téléostéens. Comp. Spermat., B. Baccetti Ed., Accademia dei Lincei, Rome, 71-79.

BILlard R.,Breton B., I970. Modifications ultrastructurales et cytochimiques des spermatozoides après dilution, chez les Poissons d'eau douce. VII e Congr. Intern. Micr. Électr. Grenoble, 637-638.

Billard R., Jalabert B., Breton B., i972. Les cellules de Sertoli des Poissons Téléostéens. I. Étude ultrastructurale. Ann. Biol. anim. Bioch. Biophys., 12, r9-32.

Drochmans P., I962. Morphologie du glycogène. Étude au microscope électronique de colora’ions négatives du glycogène particulaire. J. Ultrastruct. Res., 6, $\mathrm{r}_{4} \mathrm{I}-\mathrm{I} 6 \mathrm{r}$.

Dufaure J. P., I97I; L'ultrastructure du testicule de Lézard vivipare (Reptile, Lacertilien). II. Les cellules de Sertoli. Étude du glycogène. E. Zellforsch. ,115, 565-578.

Finck M., I960. Epoxy resins in electron microscopy. J. Biophys., Biochem. Cytol., 7, 27-3I.

Fovquer J. P., rg68. Étude infrastructurale du cycle du glycogène dans les cellules de Sertoli de Hamster. C. R. Acad. Sci., D, 267, 545-548.

Fouguet J. P., Guhs S., I969. Glycogen phosphorylase and glycogen synthetase in Hamster testis during postnatal development. $J$. Reprod. Feviti., 19, 455-464.

Georges D., I969. Spermatogenèse et spermiogenèse de Ciona intestinalis L. observées au microscope électronique. J. Microsc., 8, 39I-40o.

Giusti F., I969. The spermatozoon of a fresh-water prosobranch mollusc. J. Sibmicr. Cytol., 1, 263273.

Hochman L., I966. Reproductive properties of Coregonus lavaretus maraena (BLOCH) in pond culture. Sb. Vyo. Skoly Zem V Brnè, 150, 453-468.

Horstmann E., 1968. Die spermatozoen von Geophilus linearis кoch (Chilopoda). Z. Zellforsch., 89, $410-429$.

JALABERT B., BILlAARD R., I969. Étude ultrastructurale du site de conservation des spermatozoïdes dans l'ovaire de Poecilia reticulata (poisson téléostéen). Ann. Biol. anim. Bioch. Biophys. 9, 273-280.

LANZA B, I965. Note sugli spermatidi e gli spermi di alcuni gasteropodi glicogeno intramitochondriale e cariolinoteca. Boll, Zool., 32, I079-1086.

Lanza B., Quattrini D, I964. Osservazioni sul contenuto in glicogeno degli spermi e degli epiteli 
della gonade e delle vie sissuali ermafrodite di Vaginalus borellianus (coLosI) (Mollusca Gastropoda Sblifera). Boll. Soc. Ital. Biol. sperm., 40, II54-II55.

MANN T., 1964. Biochemistry of semen and of the male reproductive tract. Methuen, London.

Nicander L., I957. A histological study on glycogen in the testes of domestic and laboratory animals with special reference to variations during the spermatogenetic cycle. Acta Morph. Neer Scand., 1, 223-240.

Nicholls T. J., Graham G. P., I972. The ultrastructure of lobule boundary cells and Leydig cell homolog in the testis of a Cichlid Fish Cichlasoma negrofascietum. Gen. Comp. Endocr., 19, 133-146.

Personne P., André J., 1964. Existence de glycogène mitochondrial dans le spermatozoide de la Testacelle. J. Microsc., 3, 643-650.

Personne P., 1968. Existence de glycogène dans l'axonème des spermatozoïdes (abstr.). J. Microsc., 7, $50 \mathrm{a}$.

Personne P., Anderson W., r97o. Localisation mitochondriale d'enzymes liées au métabolisme du glycogène dans le spermatozoìde de l'escargot. J. Cell. Biol., 44, 20-28.

Ploye H., 1969. Sur la stabilité des portées successives chez Lebistes reticulatus. Ann. Biol. anim. Bioch. Biophys., 8, 83-89.

SANDOz D., I970. Étude cytochimique des polysaccharides au cours de la spermatogenèse d'un Amphibien Anoure : Le Discoglosse Discoglossus pictus (oтth.). J. Microsc., 9, 243-262.

Setchell B. P., Waites G. M. H., I97I. The exocrine secretion of the testis and spermatogenesis. J. Reprod. Fertil., suppl. 13, 15-27.

Silverra M., Porter K., I964. The spermatozoïds of flatworms and their microtubular systems. Protoplasma, 49, 240-265.

ThIERY J. P., 1967. Mise en évidence des polysaccharides sur coupes fines en microscopie électronique. J. Microsc., 6, 987-1018.

Von BonsDoRfF C. H., TEl.kKA A., 1965. The spermatozoon flagella of Diphylobothrium latum (fish tapeworm), Z. Zellforsch., 66, 643-648.

WINGE O., I937. Succession of broods in Lebistes. Nature, 140, 467.

\section{PLANCHE I}

\section{Localisation du glycogène au début de la spermiogenèse}

\section{A. - Dans les cellules de Sertoli.}

Au début de la spermiogenèse, les particules de glycogène apparaissent principalement dans le cytoplasme sertolien. Le cytoplasme des spermatides $s_{1 a}$ n'en contient que de faibles quantités.

$\times 28000$

\section{B. - Dans les spermatides $S_{1 b}$.}

Dès son apparition dans les spermatides rondes $S_{1 b}$ le glycogène se condense sous forme de particules $\alpha$. (double flèche) à proximité des mitochondries $m$. Des particules de glycogène $\beta$ existent en différents points du cytoplasme mais aussi dans l'espace intercellulaire $\mathrm{I}$.

$\times 28000$

Dans les deux cas : - fixation glutaraldéhyde-acide osmique ;

- coloration AP-TC-PA.

$\mathrm{m}:$ mitochondrie $\mathrm{mb}$ : membrane basale

N : noyau

$\mathrm{S}$ : cellule de Sertoli. 

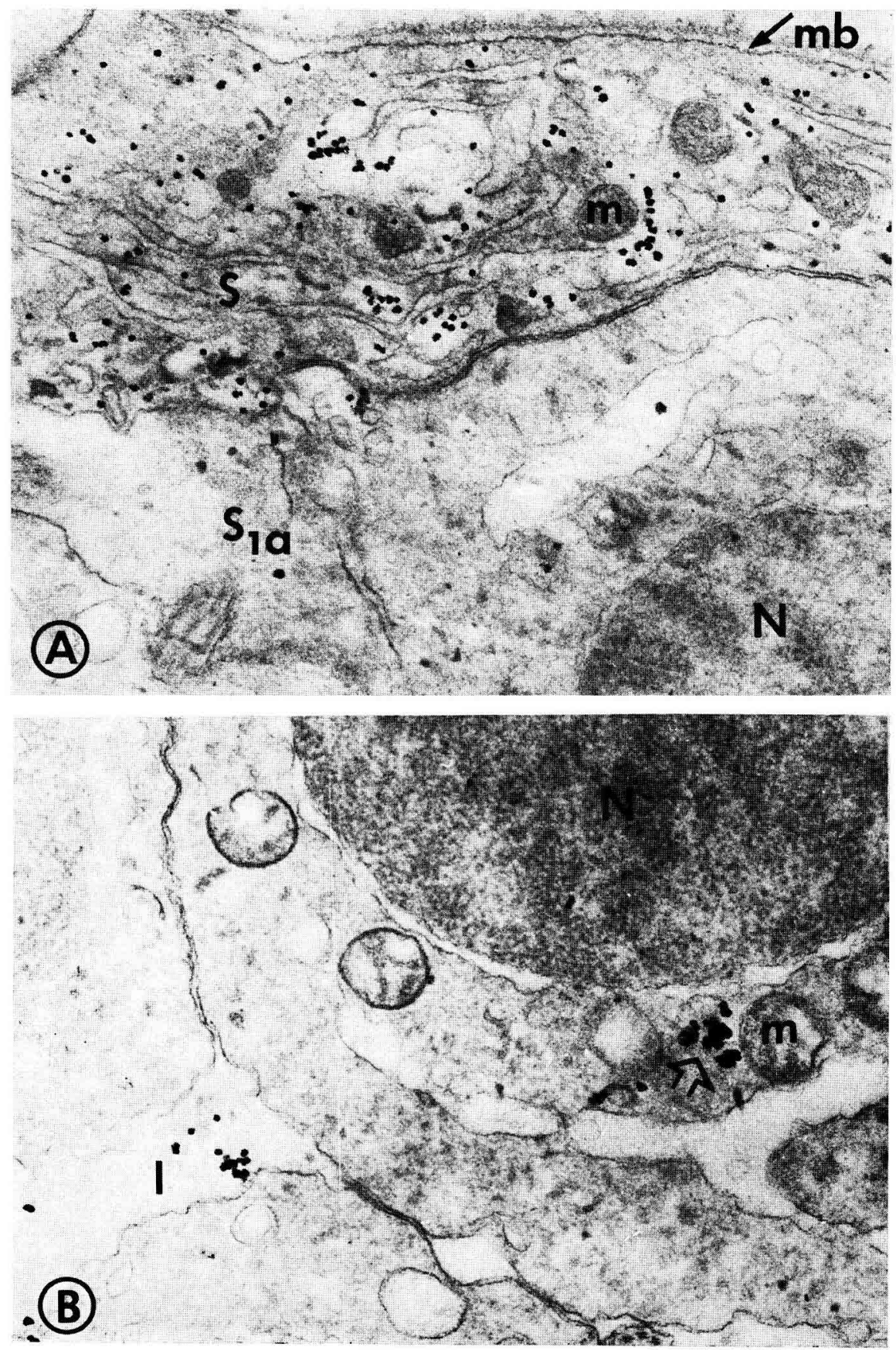

R. BILLARD et B. JALABERT 


\section{PLANCHE II}

Localisation du glycogène au niveau de la pièce intermédiaire

II A. - Spermatide $S_{1} b$.

Les particules de glycogène qui étaient localisées à proximité des mitochondries $\mathrm{m}$ (pl. I B) ont accompagné ces dernières au cours de leur migration autour du flagelle. D'autres particules de glycogène subsistent dans l'espace extracellulaire I comme dans le stade précédent (pl. I B).

$\mathrm{G}=$ appareil de Golgi ; cc $=$ complexe centriolaire. - Fixation glutaraldéhyde. - Acide osmique. - Coloration AP-TCPA. $(\times 22.500)$.

II B, C. - Spermatides $\mathrm{S}_{2}$.

B. - La taille des llots de glycogène précédemment identifiés à proximité des mitochondries a considérablement augmenté : ce glycogène se situe principalement sur la face interne du manchon mitochondrial. Les granules osmiophiles de PORTE et Follenius (1960) (flèches) qui sont particulièrement abondants à ce stade de la spermiogenèse (cf. C) ne sont pas de nature polysaccharidique. Fixation glutaraldéhyde. - Acide osmique. - Coloration APTC-PA. $(\times 28.500)$.

C. - Cette préparation a reçu une double fixation et une coloration normale uranyle-plomb. Les granules osmiophiles : go, sont particulièrement nombreux. Dans ces conditions de coloration, il est possible de retrouver les particules de glycogène (flèches). ( $\times$ 28.000). 

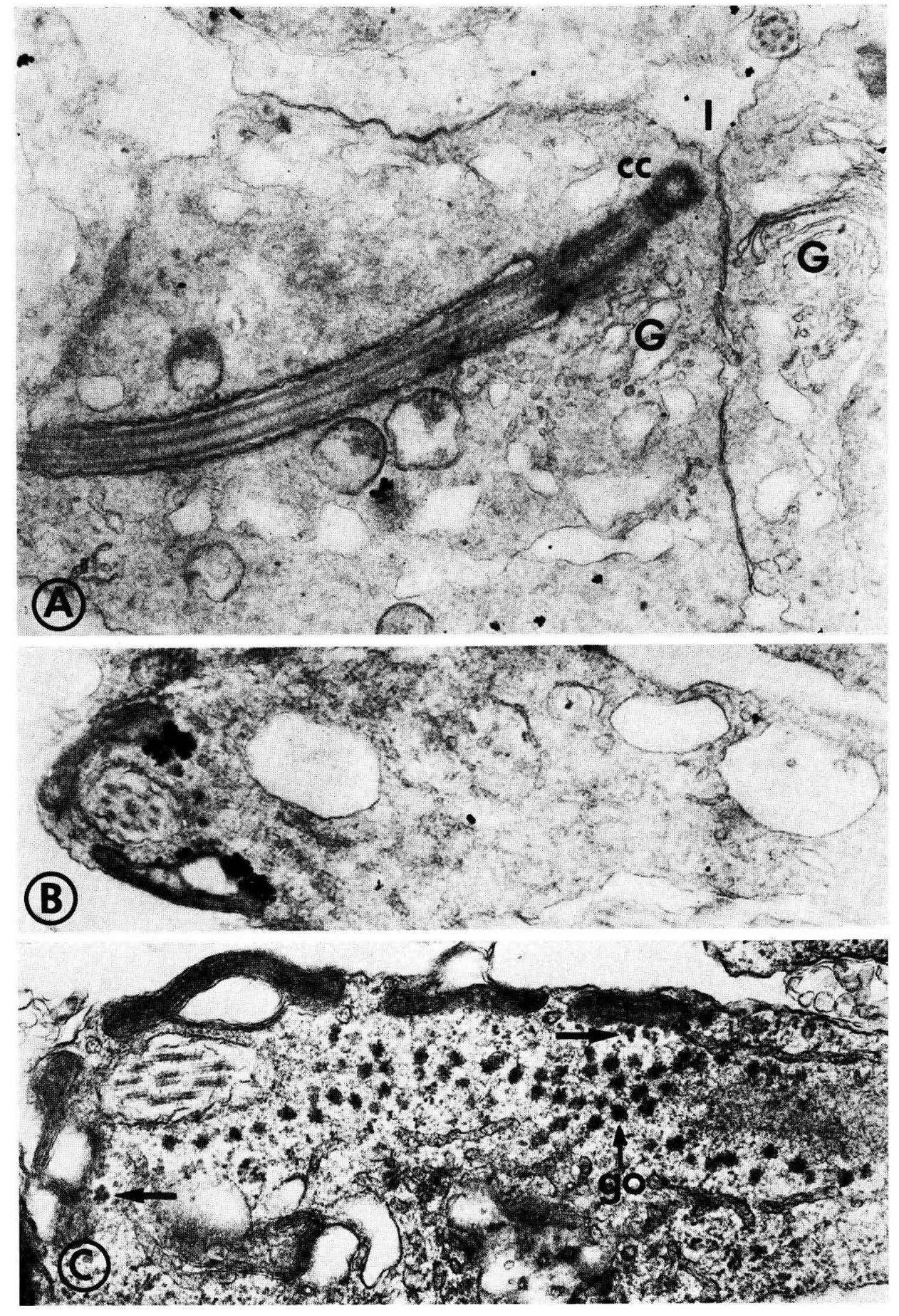


\section{PLANCHE III}

III A. - Cellules de Sertoli de cyste à spermatides $S_{2}$.

Le glycogène sous forme de particules $\beta$ est abondant dans le cytoplasme sertolien. Des particules de glycogène existent également dans la paroi des vaisseaux sanguins VS, à l'extérieur de la membrane basale.

$\mathrm{G}=$ appareil de Golgi. ( $\times 35.000)$.

III B. - Coupe longitudinale d'une spermatide $S_{2}$.

Le glycogène sous forme de particule n'est présent que dans la pièce intermédiaire de la spermatide. $(\times 22.800)$.

Dans les deux cas : Fixation glutaraldéhyde. - Acide osmique.

- Coloration AP-TC-PA. 


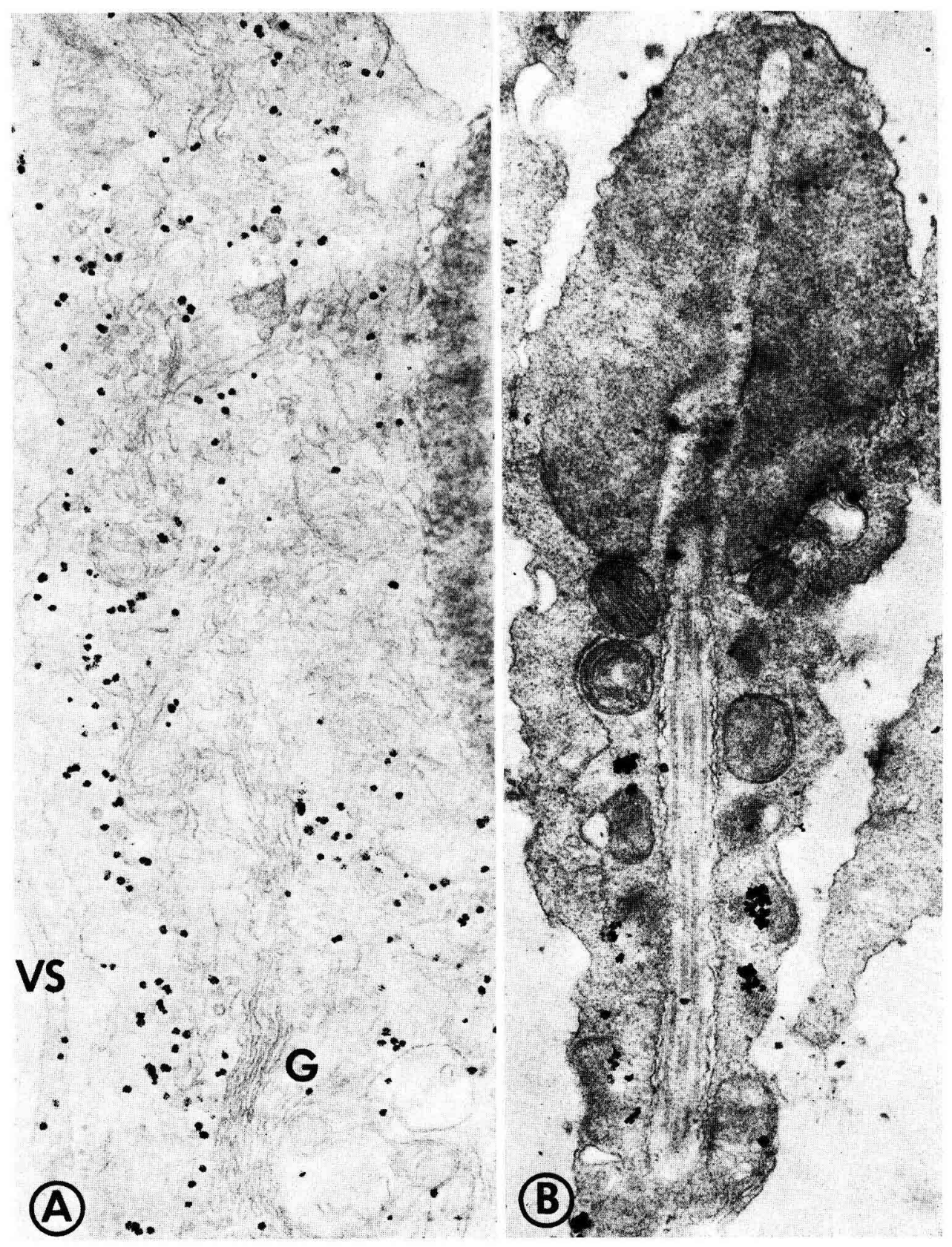

R. BILLARD et B. JALABERT 


\section{PLANCHE IV}

Le glycogène dans les spermatozoüdes du tractus génital mâle

Deux spermatozeugmes dans le canal testiculaíre : tous les spermatozoïdes du spermatozeugme situé dans la partie supérieure de la planche possèdent du glycogène, alors que dans le spermatozeugme de la partie inférieure, les spermatozoïdes en sont tous dépourvus.

Fixation glutaraldéhyde. - Acide osmique. - Coloration APTC-PA. 


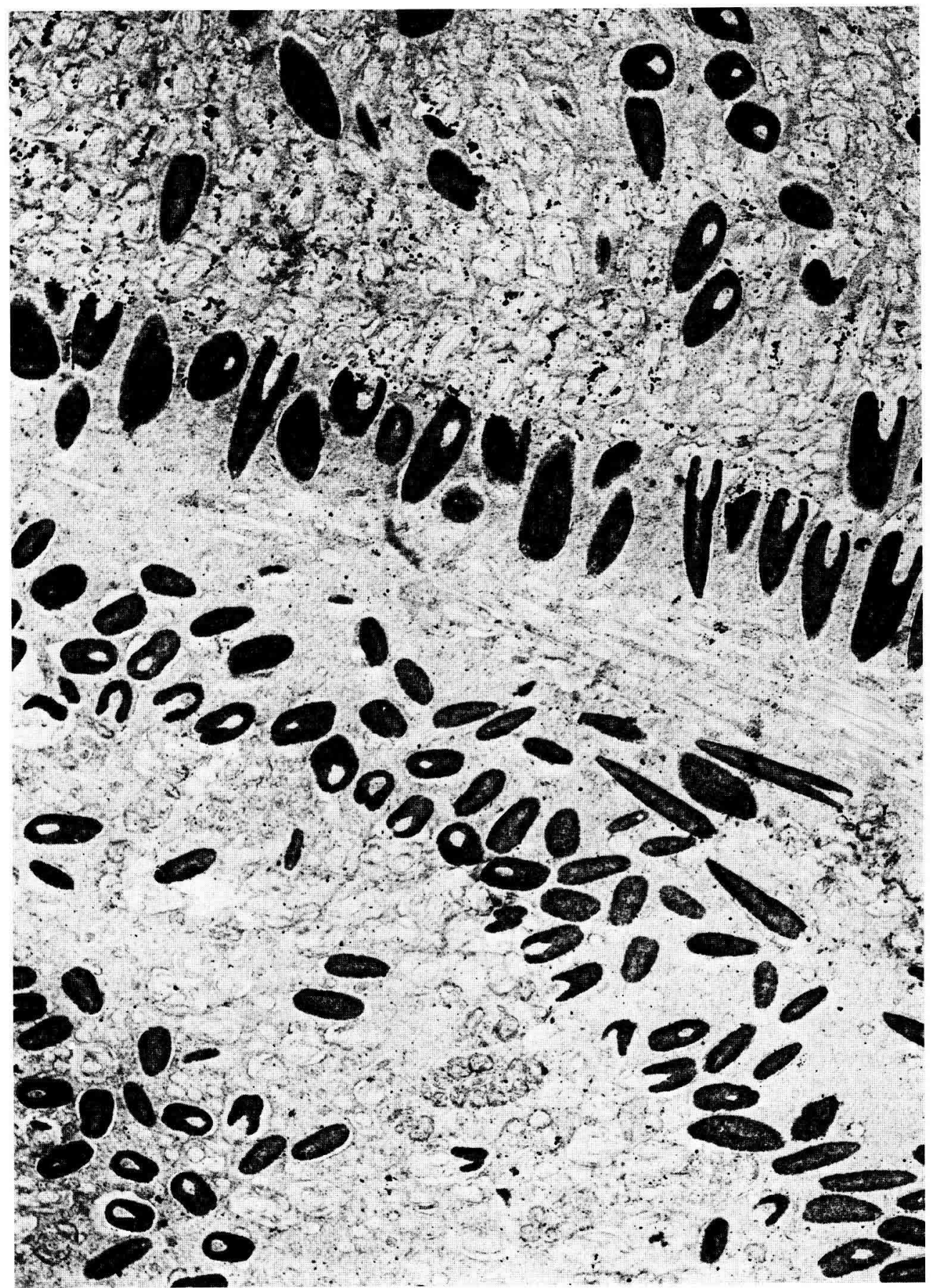




\section{PLANGHE V}

Le glycogène dans les spermatozoẗdes du tractus génital mâle (suite)

Dans les spermatozoïdes engagés dans le canal efférent, il peut subsister du cytoplasme résíduel qui n'a pas été totalement expulsé au cours de la spermiogenèse et dans lequel la réaction AP-TC-PA révèle l'existence de glycogène sous forme de particule $\alpha$.

Fixation glutaraldéhyde. - Acide osmique. - ( $\times 39.000)$. 


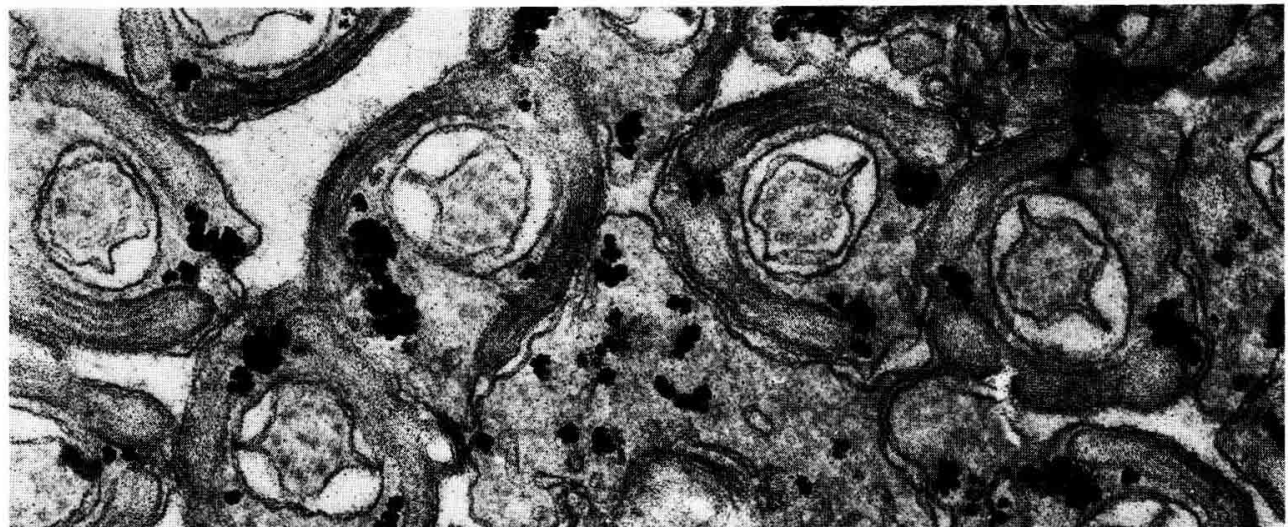

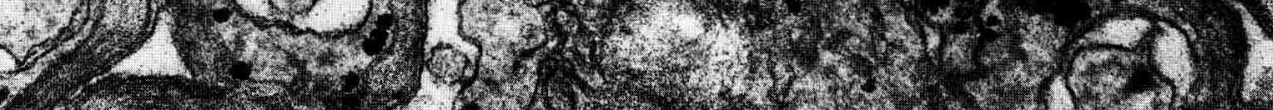

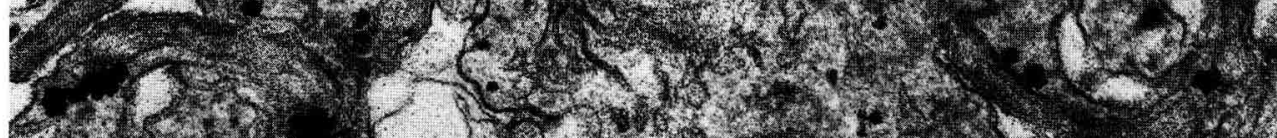
2. 2 - $2 x+2$ $218(5), 20)$

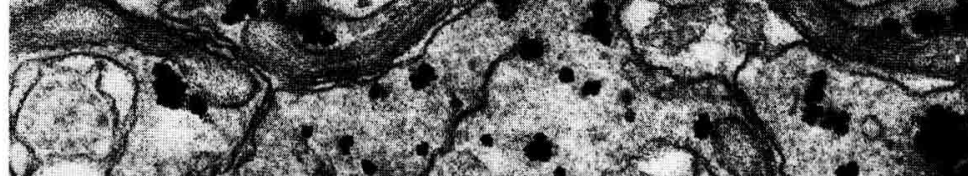

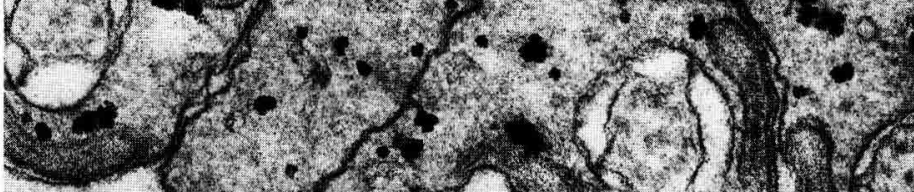

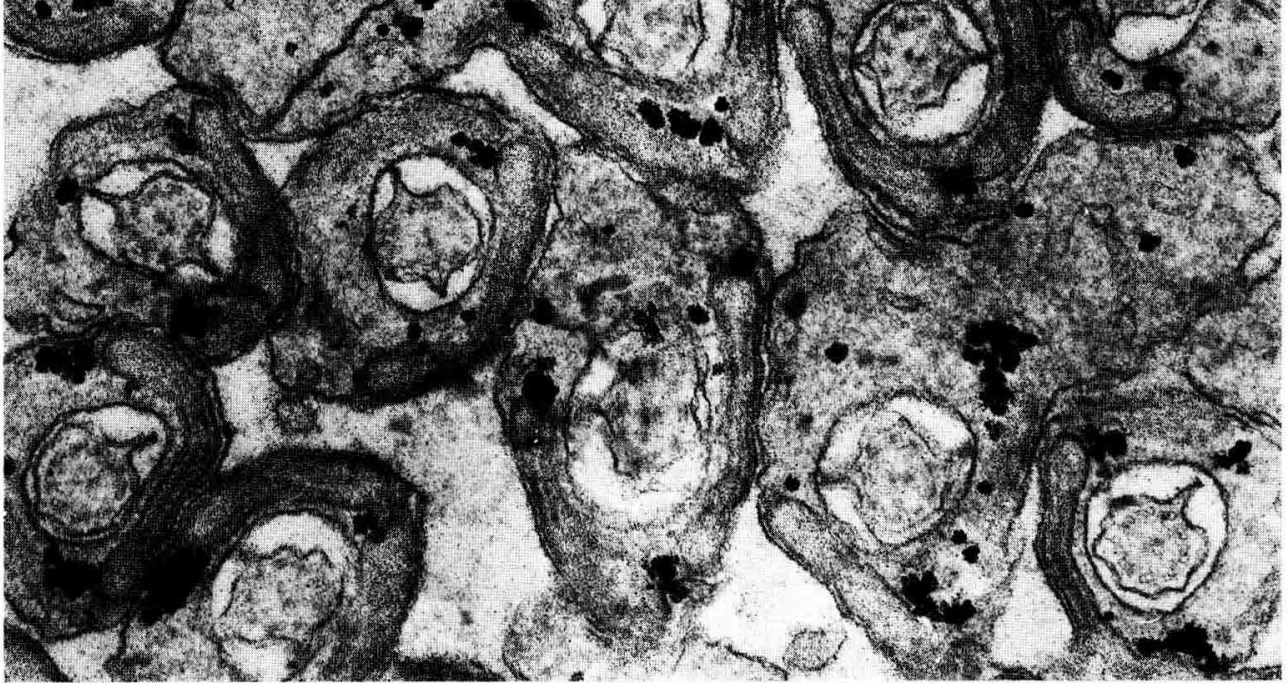

R. BILLARD et B. JALABER'T 


\section{PLANCHE VI}

Le glycogène dans les spermatozoïdes éjaculés et dans les spermatozoïdes du tractus génital femelle

Coupes longitudinales de la pièce intermédiaire de spermatozoïdes :

A. - Spermatozoïde dans le canal déférent. - Le glycogène se situe dans le manchon cytoplasmique, autour des mitochondries et du complexe centriolaire.

Fixation : glutaraldéhyde. - Coloration AP-TC-PA. $(\times 30.000)$.

B. - Spermatozoïde dans l'ovaire.

Dans ces spermatozoïdes, la quantité de glycogène est beaucoup plus faible.

Fixation : glutaraldéhyde. - Acide osmique. - Coloration AP-TC-PA. $(\times 30.000)$.

C. - Spermatozoïde éjaculé.

Fixation glutaraldéhyde. - Acide osmique. - Coloration témoin $\mathrm{N}^{\circ} 2$.

Cette coloration sans oxydation par l'acide périodique a été conduite sur une préparation postfixée à l'acide osmique. Il n'y a pas coloration des particules de glycogène mais un précipité subsiste sur les membranes. Ce précipité disparaît dans la coloration témoin No 4 . $(\times 30.000)$.

D. - Spermatozoïde éjaculé.

Fixation glutaraldéhyde. - Acide osmique. - Coloration témoin $\mathrm{N}^{\circ} 3$.

Les granules de glycogène n'apparaissent pas avec cette coloration comme avec la coloration témoin $\mathrm{N}^{\circ} 2 .(\times 30.000)$. 

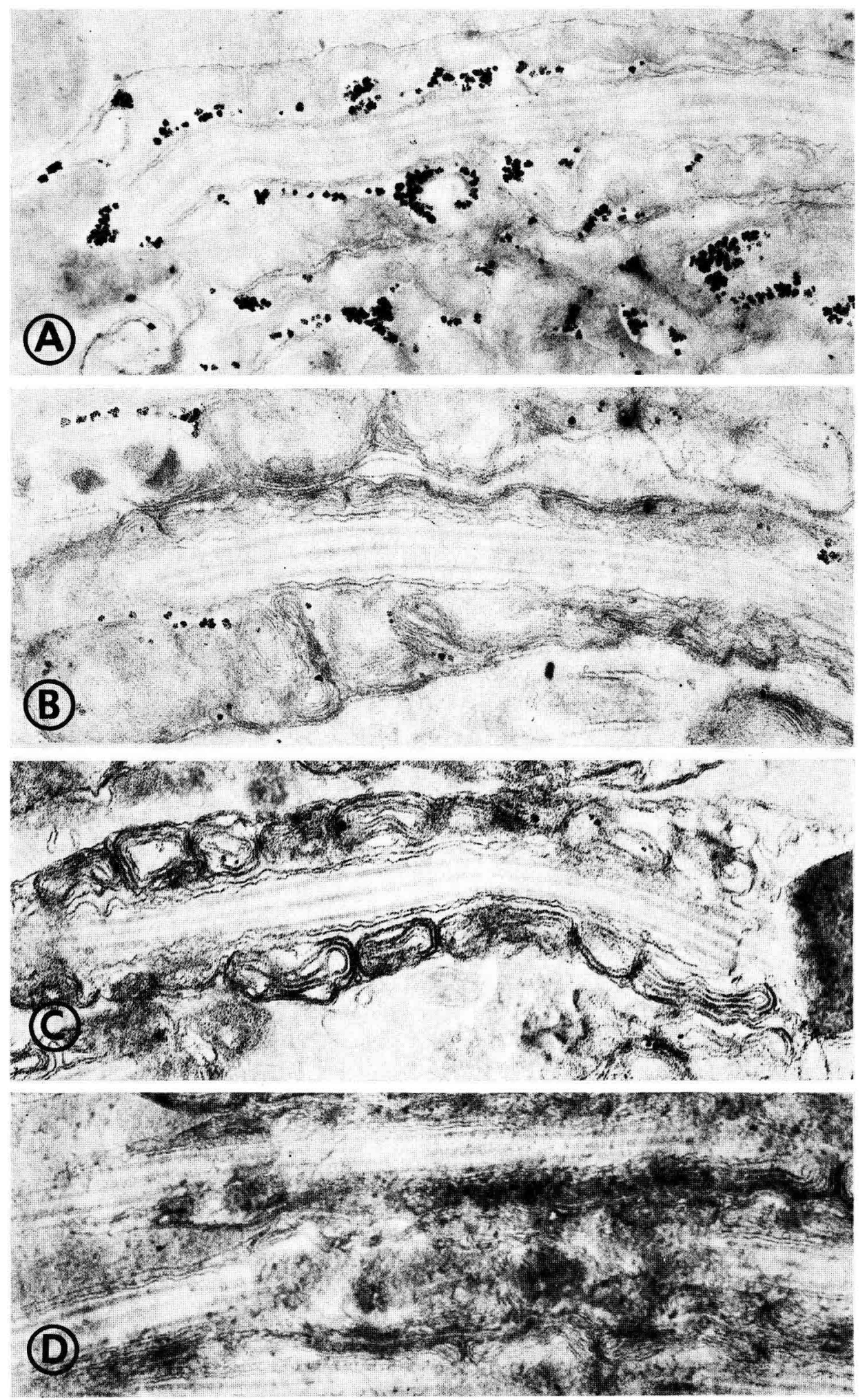


\section{PLANCHE VII}

Le glycogène dans les spermatozoïdes éjaculés

et dans les spermatozoĩdes du tractus génital femelle.

A. - Coupe transversale de pièces intermédiaires de spermatozoïdes fraîchement éjaculés. $\times 50.000$.

B. - Coupe transversale de pièces intermédiaires de spermatozoïdes implantés dans les "cellules hôtes " du réceptacle séminal dans l'ovaire. Le cytoplasme de ces cellules hôtes $c h$ qui sont des cellules épithéliales ne contient pas de particules de glycogène.

Dans les deux cas : Fixation glutaraldéhyde. - Acide osmique. - Coloration AP-TC-PA. $\times 50.000$. 

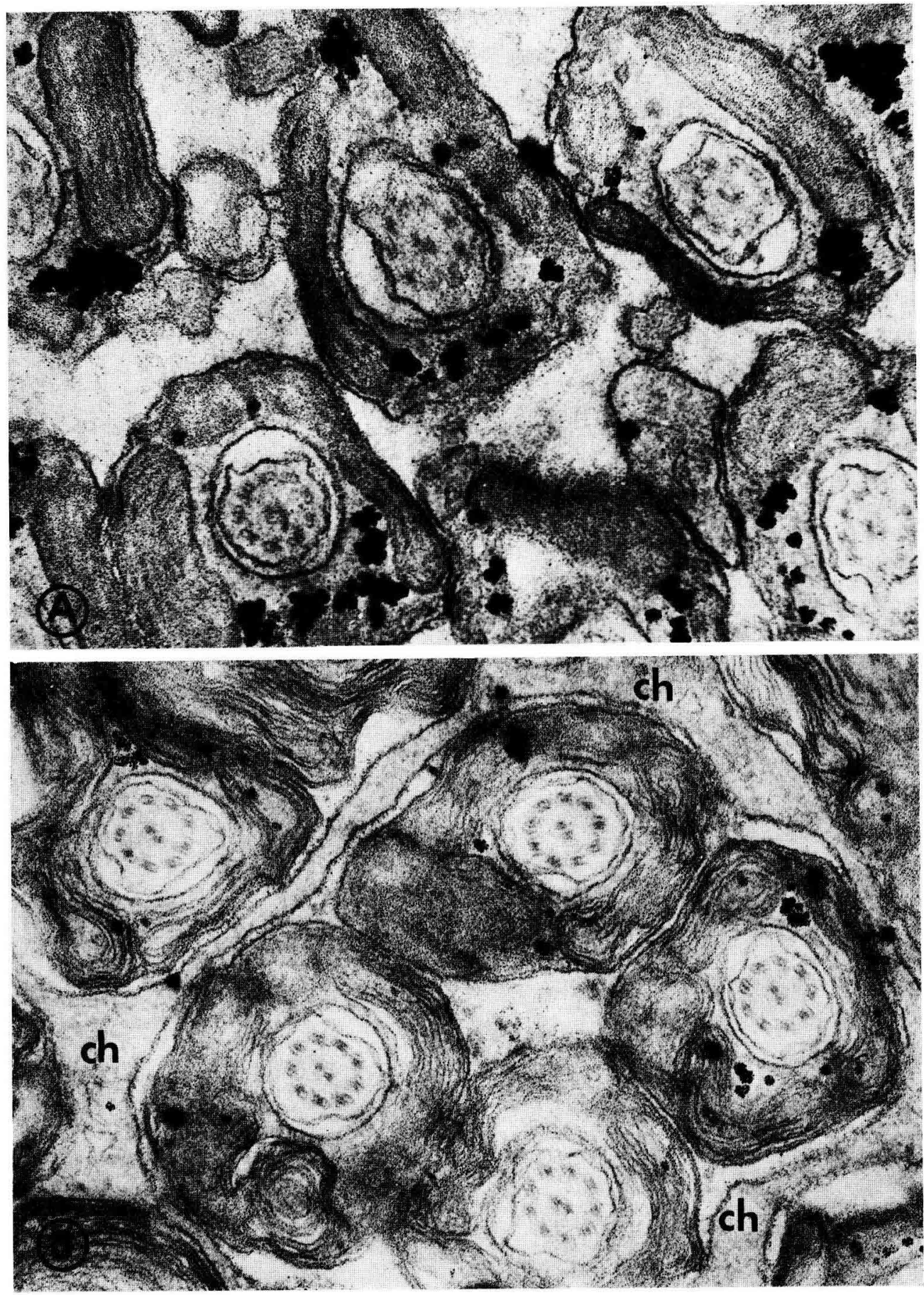

R. BILLARD et B. JALABGRT 